\title{
The Circadian Rhythm of Hormones Implications for Exercise Adaptations and Athletic Performance
}

\author{
Giorgia Scarfò ${ }^{1}$, Jonathan Fusi ${ }^{1 *}$, Marco Orsellini ${ }^{1}$, Muzaffar Asomov $^{1}$, Simona Daniele ${ }^{2}$ and Fer- \\ dinando Franzoni ${ }^{1}$ \\ ${ }^{1}$ Department of Clinical and Experimental Medicine, University of Pisa, Italy \\ ${ }^{2}$ Department of Pharmacy, University of Pisa, Italy
}

*Corresponding author: Jonathan Fusi, Department of Clinical and Experimental Medicine, University of Pisa, via Savi 10, 56126

Pisa, Italy

\section{ARTICLE INFO}

Received: 豐April 01, 2021

Published: 幽 April 08, 2021

Citation: Giorgia Scarfò, Jonathan Fusi, Marco Orsellini, Muzaffar Asomov, Simona Daniele, Ferdinando Franzoni. The Circadian Rhythm of Hormones Implications for Exercise Adaptations and Athletic Performance. Biomed J Sci \& Tech Res 35(1)2021. BJSTR. MS.ID.005640.

\begin{abstract}
The circadian Rhythm is an endogenous system that synchronizes biological processes to the natural dark/light alternation. In particular, it regulates hormone secretion creating an anabolic/catabolic environment that varies throughout the day. The circadian secretion of cortisol, testosterone, GH and other hormones influences protein synthesis and degradation, glucose metabolism, core body temperature and energy expenditure. Our aim is to review the main metabolic mechanisms regulated in a circadian manner and their implications for exercise adaptations and athletic performances.
\end{abstract}

Keywords: Circadian Rhythm; Hormones; Performance

\section{Introduction}

Life on earth undergoes recurrent changes influenced by the 24-h rotation of our planet around its axis. The earthly 24-hour axial rotation has led to the genesis and evolution of circadian clocks, that are a sort of endogenous cellular pacemakers, fundamental for organism's adaptation to the planet's light-dark rhythm [1]. It has to be noticed that these circadian rhythms are able to reproduce themselves even in the absence of external stimuli [2] because of their endogenous nature. The master circadian clock, responsible of the organism behavioral rhythm is located in the brain, although there is a molecular clock machinery in almost all cells throughout the body. In particular the site of the central circadian clock in mammals is set just above the optic chiasm: the suprachiasmatic nucleus (SCN) of the hypothalamus [3]. In mammals, the retinohypothalamic tract makes light to be the principal stimulus of the circadian clock external synchronization thanks to a direct link between the retinal innervation and the central nervous system [4].

There are also lots of clocks dispersed in our body peripheral tissues such as liver, muscle, and adipose tissue [2]. The integration of signals coming from peripheral tissues, environment, behavioral and lifestyle factors allow the central clock to regulate metabolism in a rhythmic manner [5]. SCN is able to react to internal and external stimuli sending appropriate signals to multiple endocrine axes [6]. In fact, a few studies have shown the presence of efferent projections that start in the SCN and are directed at corticotrophin-releasing hormone- (CRH) expressing neurons [7]. There is also evidence for a connection between the SCN and the somatolactotrophs (GH and somatostatin) and the reproductive axis [6]. The SCN may also influence in a circadian way the thyroid hormone release, considering that the SCN lesions cause altered T3 and T4 release [8]. Therefore, this integrated system of clocks targets a wide range of tissues and organs and the final result is an important remodulation of metabolic processes (such as insulin sensitivity and secretion, cholesterol synthesis, fat oxidation, and energy expenditure) based on the 24-hour day rhythm [2] (Figure 1). 


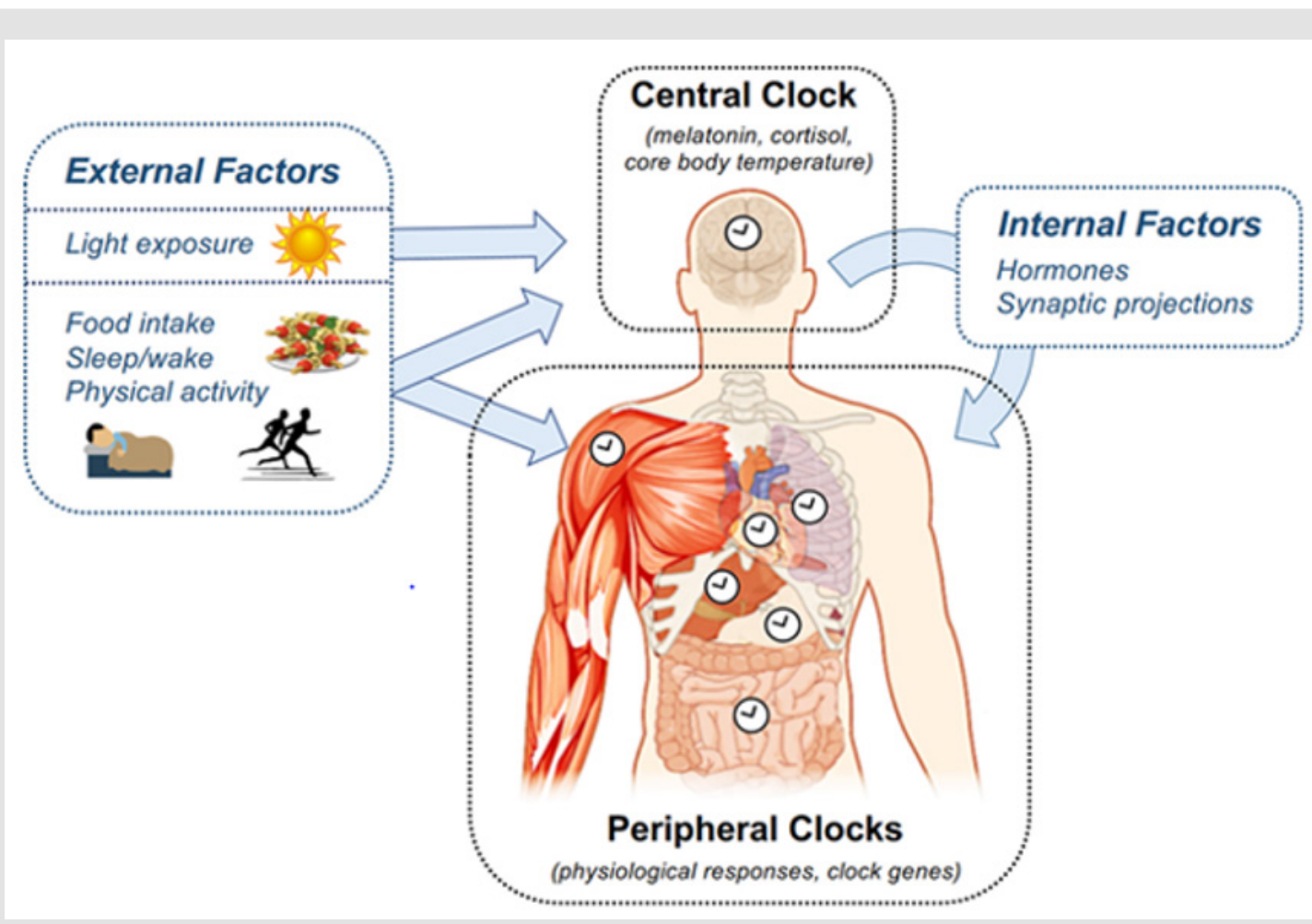

Figure 1: The Architecture of the Circadian System (Poggiogalle, 2018).

\section{Circadian Rhythm of Steroidal Hormones and Growth Hormone}

\section{Testosterone}

Testosterone influences almost every organ in the body. Its secretion is fundamental in promoting spermatogenesis, maintenance of accessory organs, muscle growth, development of secondary sexual characteristics, erythropoiesis, bone metabolism, and feedback to the hypothalamus-pituitary. Early morning total testosterone in healthy adult males is considered as normal if it ranges from approximately 300 to $1000 \mathrm{ng} / \mathrm{dL}$. Low serum levels of total testosterone or hypogonadism have several negative effects on the organism including cardiovascular disease, diabetes mellitus, low muscle mass and bone mass, low physical performance, and frailty [9]. The highest blood level is between 05.30 and $08.00 \mathrm{~h}$, while the lowest hematic level is found $12 \mathrm{~h}$ later. The variation of total $\mathrm{T}$, that is the difference between peak and mean hormone levels is about $6-12 \%$ of mean hormone level [10]. Testosterone has also positive effects on the health, lifestyle, and physical performance in a variety of subjects, both healthy and clinical ones [11].

In fact, there are evidence for a positive influence of exogenous testosterone administration on body composition, physical performance and plasma lipids in healthy young men [12]. Additionally, it has been reported a strong association between basal TES concentration and performance capacity (higher maximal vertical jump, faster $30 \mathrm{~m}$ dash and better aerobic performance) in soccer players [13]. This because Testosterone has a significant anabolic effect due to its capacity of retaining nitrogen [14], enhancing the neuromuscular transmission (Storer, 2003), and potentiating the effectiveness of other hormones including growth hormone, involved in a favorable body composition (increased FFM and decreased FM) [15].

Despite these data, it has observed an improved aerobic performance capacity also in men with lower basal $\mathrm{T}$ : in athletes trained in a similar way, ones with lower basal TES were better on the Cooper's 12-min run. In the same way, few studies showed that a reduced basal $\mathrm{T}$, was found in highly aerobically trained athletes [11]. Although SHBG has a crucial influence on circulating bioavailable TES, not many studies have focused on its crucial role in maintaining the health status [11]. Nevertheless, there are stronger connections between blood lipids and SHBG than with TES [16]. Military training studies lasting 8-12 weeks [17] have shown increased SHBG level resulting from physical training regimen. Higher SHBG levels had been also found in elite soccer players over two seasons than controls [18].

\section{Cortisol}

Cortisol is involved in several metabolic processes including glycogenolysis, lipolysis, and proteolysis. This steroidal hormone is secreted by adrenal glands. The timing of cortisol secretion is under the influence of the circadian rhythm. Its blood concentration has a peak level in the early morning, just before waking. The cortisol declines with a certain gradualness in the following hours [19]. Beside the circadian regulation, cortisol has been demonstrated to 
have an ultradian regulation that consists in a pulsatile secretion with 8-16 peaks throughout the day every 1-3 hours. Cortisol effects are visible on metabolic and neurological processes. From the metabolic point of view, it acts as a catabolic hormone by reducing lean body and muscle mass and increasing energy consumption [19].

As far as neurological processes, cortisol pulsatile secretion modulates the rhythmic expression of lots of glucorticoids-sensitive genes and helps non-genomic events, such as the postsynaptic dendritic spine formation in the cortex caused by motor skill learning. At the same time, GC circadian release is fundamental to form new spines involved in the long-term memory retention. Conversely, their chronic and excessive exposure is harmful because it could eliminate learning-associated new spines leading to the disruption of previously acquired memories [20].

\section{Growth Hormone}

Growth hormone $(\mathrm{GH})$ has the central role in the mammal growth from birth until the puberty is completed. GH is crucial in the control of body composition, somatic growth and intermediary metabolism [21]. Its secretion is subjected both to a circadian and diurnal rhythm on the trail of a sleep pattern. Specifically, GH secretion has a peak during nocturnal hours in the dark phase of the day. If this dark scheme is disrupted, there are other pulses of $\mathrm{GH}$ release during the light/awake period to compensate its possible deficiency. This means that GH release is not only under the influence of the sleep/awake cycle, but its regulation is highly complex and goes beyond the circadian rhythm [22]. GH affects metabolic processes including sleep, exercise, fasting, hypoglycaemia and hyperglycaemia. Lots of studies have shown differences between sexes: in particular we can talk about a male 'pulsatile' secretion and a female 'continuous' secretion. In addition, cortisol blood level declines with age and this phenomenon is called 'somatopause' [23].

\section{Effects of Physical Exercise on the Circadian Rhythm of Hormones}

Testosterone and Cortisol concentrations are highest in the morning and lowest in the evening, so they have a quite similar trend [24]. Despite these high blood T concentration in the early morning [25]. suggested that evening resistance training could be better for protein accretion due to an increased testosterone/ cortisol (TC) ratio. The TC ratio is a sort of anabolic/catabolic index of an organism because these two hormones have different roles in protein synthesis and protein degradation, respectively. The elevated $\mathrm{T}$ level in the morning (crucial for muscle hypertrophy) is disturbed by the morning elevated $\mathrm{C}$ level that contributes to protein degradation [26]. However, it is still unclear if exercise can exert a strong enough influence to alter the Circadian Rhythm of these hormones. In fact, studies suggest that the influences of short-term training protocols weren't able to alter the circadian rhythmicity of T and C [27]. Probably, it takes longer training period to observe an influence on the resting hormone levels [28].

As far as the superfamily of growth hormones (GH), studies suggest an association with physical activity because they are elevated during 15-30 minutes of post resistance exercise. In particular, protocols high in volume, moderate to high in intensity, using short rest intervals and stressing a large muscle mass, produce greater acute hormonal elevations (e.g. testosterone, GH and the catabolic hormone cortisol) than trainings characterized by low-volume, high-intensity protocols and long rest intervals [29]. Other anabolic hormones such as insulin and insulin-like growth factor-1 (IGF-1) are crucial for the skeletal muscle growth. Insulin secretion is influenced by blood glucose and amino acid levels. However, hematic IGF-1 increase is significant after resistance trainings probably due to GH-stimulated hepatic secretio [29].

\section{Implications for the Athletic Performance}

There are many underlying factors contributing to a Circadian Rhythm in physical performance. These factors are both internal (physiological) and external (environmental) variations that happen during the day [27]. First of all, core body temperature, with its Circadian Rhythm, is fundamental in biological processes and physical performance. There is a $0.9^{\circ} \mathrm{C}$ difference in body temperature (higher in the evening hours) and this affects muscle activity [19]. The circadian rhythm of core temperature is essential because these heating enhances metabolic reactions and the extensibility of connective tissue, reduces muscle viscosity, and improve action potential conduction velocity [30]. The increased body temperature could also lead to a better carbohydrate utilization over fat as energy substrate and promote an improvement in actinmyosin crossbridge mechanics within the musculoskeletal element [31]. In support of the positive effect of increased body temperature on physical performance [32] have found that the extension of warm-up periods during morning trainings reduced the power and force loss in countermovement jumps. In fact, additional 20 minutes of active warm-up could increase core body temperature as much as the temperature in an afternoon session. These results had already been shown by [33], who focused their attention on the relation between warm ups and cycling time trial performances.

Their results have confirmed that warm-up could improve time trial performance at both times of the day, with a mean cycling time slower at $07.30 \mathrm{~h}$ compared to $17.30 \mathrm{~h}$ even after appropriate warmups [34], investigated the performance variation in intercollegiate basketball players, in particular, player readiness, and self-reported sleep between morning and afternoon training sessions. These athletes exhibited a lower performance in the morning trainings above all in countermovement jump, power output, overall readiness, central nervous system readiness, player load and 
duration (Figure 2). Nevertheless, there is a difference between anaerobic and aerobic performances. In fact, a short-duration anaerobic performance seems to be better in the afternoon (the peak is almost always found between 16:00 and 20:00 hours) than in the morning (the peak is between 06:00 and 10:00 hours) and this could be due to the increased body temperature [35].

\begin{tabular}{lrrr}
\hline Variable & Morning & Afternoon \\
\hline CMJ (cm) & $58.8 \pm 1.3$ & $61.9 \pm 1.6$ & 0.009 \\
Power (W) & $6.378 .0 \pm 131.2$ & $6.622 .1 \pm 172.0$ & 0.009 \\
Overall readiness (AU) & $5.75 \pm 0.31$ & $5.88 \pm 0.35$ & $5.7 \pm 0.29$ \\
CNS readiness (AU) & $6.0 \pm 0.44$ & $285.4 \pm 13.9$ & 0.763 \\
PL (AU) & $275 \pm 22.9$ & $55.3 \pm 4.3$ & 0.345 \\
Duration (min) & $58.2 \pm 9.9$ & $7.4 \pm 0.25$ & 0.671 \\
Sleep (h) & $6.6 \pm 0.4$ & 0.016
\end{tabular}

$\cdot A U=$ arbitrary units.

Countermovement jump (CMJ), power output (Power), overall readiness (Overall), central nervous system readiness (CNS), Player Load (PL), and duration when comparing the morning training value with the afternoon training value with matched training intensity during the previous exposure. Data are mean $\pm S E M$.

Figure 2: Morning vs. afternoon sessions with matched training intensity and duration during the previous exposure (Heishman, 2017).

As far as long-duration aerobic exercises, the effects of the circadian regulation could be equivocal [36], have suggested that there is a circadian specificity in an aerobic training to improve the anaerobic threshold: after 6 weeks of training, the ventilatory anaerobic threshold was higher in the morning in athletes who had trained in the morning hours, while it was higher in the afternoon in those who had trained in the afternoon. In the control group, there were no threshold fluctuations throughout the day, but they concluded that exercise adaptations were probably better if the aerobic training was performed in the afternoon. In contrast [37] clearly have shown that exercise aerobic performance is greater in the morning ( $06.45 \mathrm{~h}$ ) if the training was performed in a warm environment. Finally, it is important to reflect on the strength training: several studies have shown that the adaptation to strength training depends on when which training was performed.

For example [37], focused on the effects of training at the same time of the day on the diurnal variations of anaerobic performances to in order to adjust training hours with the time of the day of competitive events. Thirty athletes performed a lower-extremity progressive resistance training 3 times per week for 8 weeks and this training was designed jest to improve muscular strength and power. These subjects were randomly divided into a morning training group (MTG, 07:00-08:00 hours, $\mathrm{n}=10$ ), an evening training group (ETG, 17:00-18:00 hours, $\mathrm{n}=10$ ), and a control group (CG, who underwent all tests but didn't train, $n=10$ ). Before and after 2 week and 8week regular training, it was analyzed the ability in the squat jump, the countermovement jump, the Wingate and 1 repetition maximum (1RM) during leg extension, leg curl, and squat tests was. For all the participants, the morning and evening tests were performed at the same time of the day as for the morning and evening training sessions.

Before training, the results indicated a significant increase in performance from morning to evening tests (ca. 2.84-17.55\% for all tests) for all groups. After training, the diurnal variations in anaerobic performances were not so significant in the MTG. In fact, there was no important difference in muscular power or strength between morning and evening tests but these circadian variations in anaerobic performances was still substantial in the ETG and CG. In a few words, adaptations to strength training are ideal at the time of the day at which training was performed.

\section{Meal Planning and Energy Expenditure}

Regardless of mealtimes and physical activities, glucose metabolism has its own circadian rhythm. In particular, it has reported the existence of a diurnal rhythm in oral glucose tolerance, with a typical morning peak and a weakening in glucose tolerance in the afternoon and evening [2]. Insulin is the most important hormone involved in blood glucose levels control. Both secretion of insulin from the pancreas and the systemic insulin sensitivity follow a circadian pattern [38]. On one hand, feeding is not essential for rhythmic glucose or insulin regulation in the normal physiological condition because a study suggests that 6 identical meals per day did not alter rhythmic glucose or insulin responses [39]. On the other hand, food can cause a substantial modulation in the peripheral circadian clocks [40]. Limiting feeding only at the light phase almost could eliminate high fat diet-induced metabolic storm even in absence of a total calorie intake change. Conversely, feeding in the sleep phase perturbed the metabolism greatly [41].

Eating in late evening is considered as a risk factor for a negative metabolic array causing an increased fat mass and BMI [42]. Besides, an intense evening training, without an important reintegration of carbs and the decrease in glucose level, stimulates lipid oxidation in muscles fibers [43]. Several studies suggested also that the individual energy expenditure has its own circadian rhythm. One of them, using indirect calorimetry in 7 healthy young men, has reported a $17 \%$ change in energy expenditure, with highest levels at 9.00 am and 12.00 and lower ones between 24.00 and 6.00 am [44]. A second study has focused on oxygen 
consumption and carbon dioxide production in 10 healthy young men suggesting a variation of $6 \%$ in these parameters [45]. Both trials reported no significant circadian variation in the respiratory quotient (RQ), which is an index of which substrate they were using [45]. Another trial on 15 obese adults, fasting 24 hours, showed that the energy expenditure was better in the afternoon (between 13.15 and $17.23 \mathrm{~h}$ ) [46].

There is also a difference in the postprandial energy expenditure. In particular, food has a variation in its thermic effect that is up to $44 \%$ higher in the morning comparing to the afternoon and evening [47]. In any case the greatest use of glycogen and fatty acids happens under fasting states in order to improve weight loss. Therefore, it is essential that training is planned in relation to meals to improve performance and respect individual circadian rhythms.

\section{Conclusion}

Considering the above, it is possible to create training programs indulging the biological circadian rhythm in order to improve performances and build better physiological assumption for the adaptation. It has been noticed, for example, that morning trainings cannot render, in terms of intensity, as much as afternoon ones. It is also true that the morning cortisol peak is able to stimulate the dendritic remodeling suggesting that in the early morning could be useful an activity based on learning new motor skills paying attention to recovery times in order to restore the nervous system. A fasting aerobic workout instead, could be useful to lose weight. In fact, as it has described, on waking up, there is a glycemic elevation (without any food intake) to avoid hypoglycemic crises during training.

At the same time, an absence of pre-training food intake stimulates the lipid degradation even though this condition could alter the glycemic control and impoverish liver and muscle glycogen, nullifying both the training and the recovery. As far as strength training, a several-week morning workout could improve this capacity to the point that the athlete is able to have great strength performances regardless of the time. The most important thing is maintaining a lower intensity level during exercises above all in subjects with cardiovascular risk factors. This because, on waking up, there is the shift from a vagal to a sympathetic tone that could be a trigger for cardiovascular issues [48]. It is evident instead, that afternoon is the best moment to train: it is possible to perform all types of training without any contraindications and with excellent results. In particular, most suitable afternoon exercises are:

a) High-medium intensity aerobic workouts.

b) Strength and hypertrophy exercises.

c) High intensity circuit training.

Concerning evening training, it is important to focus on the sleep/wake rhythm: dark hour physical activity could affect the melatonin rhythm in a negative way and so alter the sleep quality [49-51]. It should be performed only a low-impact aerobic activity. Obviously, these training directions must take into account that gene expression influences adaptation to training and food and this genetic variability conditions performance enormously.

\section{References}

1. Husse J, Eichele G, Oster H (2015) Synchronization of the mammalian circadian timing system: Light can control peripheral clocks independently of the SCN clock: Alternate routes of entrainment optimize the alignment of the body's circadian clock network with external time. BioEssays 37(10): 1119-1128.

2. Poggiogalle E, Jamshed H, Peterson CM (2018) Circadian regulation of glucose, lipid, and energy metabolism in humans. Metabolism 84: 11-27.

3. Hastings MH, Maywood ES, Brancaccio M (2019) Suprachiasmatic nucleus in the mouse: Retinal innervation, intrinsic organization and efferent projections. Biology (Basel) 916(1-2): 172-191.

4. Abrahamson EE, Moore RY (2001) Suprachiasmatic nucleus in the mouse: Retinal innervation, intrinsic organization and efferent projections. Brain Res (1-2): 172-191.

5. Barclay JL, Tsang AH, Oster H (2012) Interaction of central and peripheral clocks in physiological regulation. In: Progress in Brain Research 199: 163-181.

6. Tonsfeldt KJ, Chappell PE (2012) Clocks on top: The role of the circadian clock in the hypothalamic and pituitary regulation of endocrine physiology. Mol Cell Endocrinol 349(1): 3-12.

7. Buijs RM, Markman M, Nunes Cardoso B, Hou YX, Shinn S (1993) Projections of the suprachiasmatic nucleus to stress-related areas in the rat hypothalamus: A light and electron microscopic study. J Comp Neurol 335(1): 42-54.

8. Kalsbeek A, Fliers E, Franke AN, Wortel J, Buijs RM (2000) Functional connections between the suprachiasmatic nucleus and the thyroid gland as revealed by lesioning and viral tracing techniques in the rat. Endocrinology 141: 3832-3841.

9. González-Sales M, Barrière O, Tremblay PO, Nekka F, Desrochers J, et al. (2016) Modeling Testosterone Circadian Rhythm in Hypogonadal Males: Effect of Age and Circannual Variations. AAPS J.

10. Brambilla DJ, Matsumoto AM, Araujo AB, McKinlay JB (2009) The effect of diurnal variation on clinical measurement of serum testosterone and other sex hormone levels in men. J Clin Endocrinol Metab 94(3): 907913.

11. Gagnon SS, Nindl BC, Vaara JP, Santtila M, Häkkinen K, et al. (2018) Basal endogenous steroid hormones, sex hormone-binding globulin, physical fitness, and health risk factors in young adult men. Front Physiol.

12. Bhasin S, Woodhouse L, Casaburi R (2001) Testosterone dose-response relationships in healthy young men. Am J Physiol Endocrinol Metab.

13. Bosco C, Tihanyi J, Viru A (1996) Relationships between field fitness test and basal serum testosterone and cortisol levels in soccer players. Clin Physiol 16(3): 317-322.

14. Storer TW, Magliano L, Woodhouse L, Richard Casaburi, Shalender Bhasin, et al. (2003) Testosterone dose-dependently increases maximal voluntary strength and leg power but does not affect fatigability or specific tension. J Clin Endocrinol Metab 88(4): 1478-1485.

15. Giustina A, Veldhuis JD (1998) Pathophysiology of the Neuroregulation of Growth Hormone Secretion in Experimental Animals and the Human. Endocr Rev.

16. Bataille V, Perret B, Evans A (2005) Sex hormone-binding globulin is a major determinant of the lipid profile: The PRIME study. Atherosclerosis 172(2): 369. 
17. Tanskanen MM, Kyröläinen H, Uusitalo AL (2011) Serum sex hormone binding globulin and cortisol concentrations are associated with overreaching during strenuous military training. J Strength Cond Res 25(3): 787-797.

18. Hammami MA, Ben Abderrahman A, Hackney AC (2017) Hormonal (Cortical-Gonadotropic Axis) and Physical Changes with Two Years Intense Exercise Training in Elite Young Soccer Players. J Strength Cond Res 31(9): 2388-2397.

19. Serin Y, Acar Tek N (2019) Effect of Circadian Rhythm on Metabolic Processes and the Regulation of Energy Balance. Ann Nutr Metab.

20. Kalafatakis K, Russell GM, Lightman SL (2019) Mechanisms in endocrinology: Does circadian and ultradian glucocorticoid exposure affect the brain? Eur J Endocrinol 180(2): R73-R89.

21. Kato Y, Murakami Y, Sohmiya M, Nishiki M (2002) Regulation of human growth hormone secretion and its disorders. Intern Med 41(1): 7-13.

22. Vakili H, Jin Y, Cattini PA (2016) Evidence for a circadian effect on the reduction of human growth hormone gene expression in response to excess caloric intake. J Biol Chem (26): 13823-13833.

23. Lim CT, Khoo B (2000) Normal Physiology of Acth.

24. Hatfield DL, Nicoll JX, Kraemer WJ (2016) Effects of circadian rhythm on power, force, and hormonal response in young men. J Strength Cond Res 30(3): 725-732.

25. Deschenes MR, Kraemer WJ, Bush JA (1998) Biorhythmic influences on functional capacity of human muscle and physiological responses. Med Sci Sports Exerc 30(9): 1399-1407.

26. Hayes LD, Bickerstaff GF, Baker JS (2010) Interactions of cortisol, testosterone, and resistance training: Influence of circadian rhythms. Chronobiol Int 27(4): 675-705.

27. Teo W, Newton MJ, McGuigan MR (2011) Circadian rhythms in exercise performance: Implications for hormonal and muscular adaptation. J Sport Sci Med 10(4): 600-606.

28. Sedliak M, Finni T, Cheng S, Kraemer WJ, Häkkinen K (2007) Effect of time-of-day-specific strength training on serum hormone concentrations and isometric strength in men. Chronobiol Int pp. 1159-1177.

29. Kraemer WJ, Ratamess NA (2005) Hormonal responses and adaptations to resistance exercise and training. Sport Med 35: 339-361.

30. Chtourou H, Driss T, Souissi S, Gam A, Chaouachi A, et al. (2012) The effect of strength training at the same time of the day on the diurnal fluctuations of muscular anaerobic performances. J Strength Cond Res 26(1): 217-225.

31. Starkie RL, Hargreaves M, Lambert DL, Proietto J, Febbraio MA (1999) Effect of temperature on muscle metabolism during submaximal exercise in humans. Exp Physiol.

32. Taylor K, Cronin JB, Gill N, Chapman DW, Sheppard JM (2011) Warm-up affects diurnal variation in power output. Int J Sports Med 32(3): 185189.

33. Atkinson G, Todd C, Reilly T, Waterhouse J (2005) Diurnal variation in cycling performance: Influence of warm-up. J Sports Sci.

\section{ISSN: 2574-1241}

DOI: $10.26717 /$ BJSTR.2021.35.005640

Jonathan Fusi. Biomed J Sci \& Tech Res

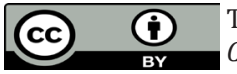

This work is licensed under Creative Commons Attribution 4.0 License

Submission Link: https://biomedres.us/submit-manuscript.php
34. Heishman AD, Curtis MA, Saliba EN, Hornett RJ, Malin SK, et al. (2017) Comparing performance during morning vs. afternoon training sessions in intercollegiate basketball players. J Strength Cond Res (6): 1557-1562.

35. Chtourou H, Souissi N (2012) The effect of training at a specific time of day: A review. J Strength Cond Res.

36. Hill DW, Leiferman JA, Lynch NA, Dangelmaier BS, Burt SE (1998) Temporal specificity in adaptations to high-intensity exercise training. Med Sci Sports Exerc 30(3): 450-455.

37. Hobson RM, Clapp EL, Watson P, Maughan RJ (2009) Exercise capacity in the heat is greater in the morning than in the evening in man. Med Sci Sports Exerc 41(1): 174-180.

38. Kitazawa M (2013) Circadian rhythms, metabolism, and insulin sensitivity: Transcriptional networks in animal models. Curr Diab Rep.

39. Kalsbeek A, Strubbe JH (1998) Circadian control of insulin secretion is independent of the temporal distribution of feeding. Physiol Behav 63(4): 553-558.

40. Tognini P, Murakami M, Liu Y (2017) Distinct Circadian Signatures in Liver and Gut Clocks Revealed by Ketogenic Diet. Cell Metab 26(3): 523538.

41. Hatori M, Vollmers C, Zarrinpar A (2012) Time-restricted feeding without reducing caloric intake prevents metabolic diseases in mice fed a high-fat diet. Cell Metab 15(6): 848-860.

42. Challet E (2019) The circadian regulation of food intake. Nat Rev Endocrinol.

43. Gabriel BM, Zierath JR (2019) Circadian rhythms and exercise re-setting the clock in metabolic disease. Nat Rev Endocrinol.

44. Krauchi K, Wirz Justice A (1994) Circadian rhythm of heat production, heart rate, and skin and core temperature under unmasking conditions in men. Am J Physiol - Regul Integr Comp Physiol (3 Pt 2): R819-829.

45. Spengler CM, Czeisler CA, Shea SA (2000) An endogenous circadian rhythm of respiratory control in humans. J Physiol 526(3): 683-694.

46. Consoli A, Capani F, Del Ponte A, G Ditano, S Sensi, et al. (1981) Effect of scheduling of mealtimes on the circadian rhythm of energy expenditure. Boll Soc Ital Biol Sper 57(23): 2322-2344.

47. Zwiauer KFM, Mueller T, Widhalm K (1992) Effect of daytime on resting energy expenditure and thermic effect of food in obese adolescents. J Am Coll Nutr pp. 267-271.

48. Black N, D’Souza A, Wang Y (2019) Circadian rhythm of cardiac electrophysiology, arrhythmogenesis, and the underlying mechanisms. Hear Rhythm 16(2): 298-307.

49. Barger LK, Wright KP, Hughes RJ, Czeisler CA (2004) Daily exercise facilitates phase delays of circadian melatonin rhythm in very dim light. Am J Physiol Regul Integr Comp Physiol.

50. Bhasin S, Woodhouse L, Storer TW (2003) Androgen effects on body composition. Growth Horm IGF Res 13: S63-S71.

51. Gerhart Hines Z, Lazar MA (2015) Circadian metabolism in the light of evolution. Endocr Rev 36: 289-304.

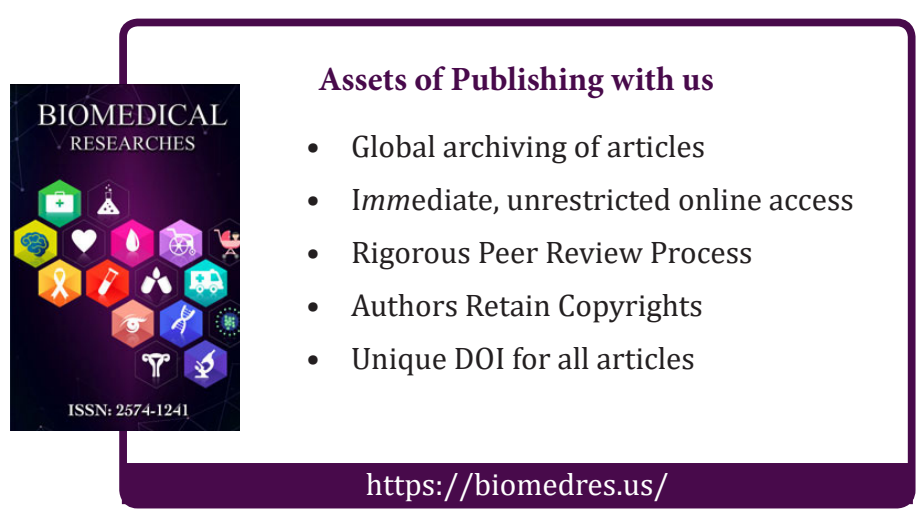

